\title{
Urinary phytoestrogens and cancer, cardiovascular, and all-cause mortality in the continuous National Health and Nutrition Examination Survey
}

\author{
Michael K. Reger ${ }^{1,2}$, Terrell W. Zollinger ${ }^{1}$, Ziyue Liu ${ }^{3}$, Josette Jones ${ }^{4}$, and Jianjun Zhang ${ }^{1,5}$ \\ ${ }^{1}$ Department of Epidemiology, Indiana University Richard M. Fairbanks School of Public \\ Health, Indianapolis, IN \\ ${ }^{2}$ College of Health Professions, Ferris State University, Big Rapids, MI* \\ ${ }^{3}$ Department of Biostatistics, Indiana University Richard M. Fairbanks School of Public Health \\ and School of Medicine, Indianapolis, IN \\ ${ }^{4}$ Department of Health Informatics, School of Informatics and Computing, Indiana University- \\ Purdue University Indianapolis, Indianapolis, IN \\ ${ }^{5}$ Indiana University Melvin and Bren Simon Cancer Center, Indianapolis, IN
}

* Present address for Michael K. Reger.

\author{
Address for correspondence and reprints: \\ Jianjun Zhang, MD, PhD \\ Department of Epidemiology \\ Indiana University Fairbanks School of Public Health \\ 714 N Senate Avenue, Suite EF250F \\ Indianapolis, IN 46202 \\ Phone: (317) 274-4287 \\ Fax: (317) 274-3443 \\ Email: JZ21@iu.edu
}

This is the author's manuscript of the article published in final edited form as:

Reger, M. K., Zollinger, T. W., Liu, Z., Jones, J., \& Zhang, J. (2015). Urinary phytoestrogens and cancer, cardiovascular, and all-cause mortality in the continuous National Health and Nutrition Examination Survey. European journal of nutrition, 1-12. http://dx.doi.org/10.1007/s00394-015-0917-y 


\begin{abstract}
Purpose: Experimental studies suggest that phytoestrogen intake alters cancer and cardiovascular risk. This study investigated the associations of urinary phytoestrogens with total cancer $(n=79)$, cardiovascular $(n=108)$, and allcause $(n=290)$ mortality among 5,179 participants in the continuous National Health and Nutrition Examination Survey (1999-2004).
\end{abstract}

Methods: Urinary phytoestrogens were measured using high performance liquid chromatography with tandem mass spectrometric detection. Survival analysis was performed to evaluate hazard ratios (HRs) and 95\% confidence intervals (CIs) for each of the three outcomes in relation to urinary phytoestrogens.

Results: After adjustment for confounders, higher urinary concentrations of total enterolignans were associated with a reduced risk of death from cardiovascular disease (HR for tertile 3 vs. tertile 1: 0.48; 95\% CI: 0.24, 0.97), whereas higher urinary concentrations of total isoflavones (HR for tertile 3 vs. tertile 1: 2.14; 95\% CI: 1.03, 4.47) and daidzein (HR for tertile 3 vs. tertile 1: 2.05; 95\% CI: 1.02, 4.11) were associated with an increased risk. A reduction in all-cause mortality was observed for elevated urinary concentrations of total enterolignans (HR for tertile 3 vs. tertile 1: 0.65; 95\% CI: 0.43, 0.96) and enterolactone (HR for tertile 3 vs. tertile 1: 0.65; 95\% CI: 0.44, 0.97).

Conclusions: Some urinary phytoestrogens were associated with cardiovascular and all-cause mortality in a representative sample of the U.S. population. This is one of the first studies that used urinary phytoestrogens as biomarkers of their dietary intake to evaluate the effect of these bioactive compounds on the risk of death from cancer and cardiovascular disease.

Keywords: Cancer; cardiovascular disease; cohort study; mortality; urinary phytoestrogens

\title{
Introduction
}

Cardiovascular disease and cancer are the leading causes of death in the United States [1] and many other developed countries throughout the world [2]. In the United States, 597,689 cardiovascular deaths and 574,743 cancer deaths occurred in 2010 [1]. On a global scale, cardiovascular disease was estimated to account for over 13.2 million deaths in 2011 [2], and total cancers claimed an estimated 8.2 million lives in 2012 [3]. To prevent the 
development of cancer and cardiovascular disease, it is necessary to identify their risk factors, particularly modifiable ones. One such modifiable factor is diet.

Phytoestrogens are a group of non-steroidal plant metabolites. The principal classes of phytoestrogens include isoflavones and lignans. Isoflavones abound in soy products, legumes, and chick peas [4,5], and lignans primarily originate from seed oils, whole grain cereals, and beans [6]. Isoflavones found in soy products include genistein, daidzein, and glycitein [7], with these compounds arising after metabolism by the gut bacteria of the glycoside conjugates [8]. Daidzein can be further converted into two endogenous metabolites, equol and Odesmethylangolensin, with individual variation in the metabolism of daidzein in populations $[9,10]$. Lignans commonly consumed by humans include enterolactone and enterodiol [11]. Differences in the biochemistry and food sources of individual phytoestrogens requires investigation of both the overall effect of total phytoestrogens as a single family of bioactive compounds and the independent effect of each phytoestrogen in relation to disease risk.

A growing body of experimental evidence suggests that it is biologically plausible that phytoestrogen intake may modulate the risk of cancer and cardiovascular disease [12-15]. Phytoestrogens can induce biologic responses due to their structural similarity to $17 \beta$-estradiol when they are consumed in the diet [16]. The biologic responses from phytoestrogens include estrogenic, anti-estrogenic, anti-oxidative, anti-viral, anti-bacterial, and antiproliferative effects [11]. It has been found that the potential beneficial effect of phytoestrogens on some hormonerelated cancers $[17,18]$ is mediated through their competitive binding to estrogen receptors $[19,20]$. While estradiol exhibits an equal affinity to both $\alpha$ and $\beta$ receptors (ER $\alpha$ and $E R \beta)$, phytoestrogens show a stronger affinity to ER $\beta$ [21]. For example, genistein has an approximately 30 -fold greater affinity to the ER $\beta$, and therefore may cause some clinical effects by selectively triggering this particular receptor [21]. Administration of phytoestrogens reduced serum testosterone levels in rats, an established risk factor for prostate cancer [12,22]. It was also found that soy phytoestrogens reversed severe pulmonary hypertension and prevented heart failure in the same animals [13].

Despite experimental evidence, few epidemiologic studies have examined the associations between phytoestrogen intake and cancer or cardiovascular mortality in Western populations. Previous studies have focused on a few sites of cancer, mainly prostate $[23,24]$ and breast $[25,26]$, yielding mixed results. Little is known about the association between phytoestrogen intake and cardiovascular disease [27], although it is considered a promising area of research for cardiovascular disease prevention [28]. The consumption of soy products is lower in Western 
countries than in Asian countries [18,29]. However, several studies have reported a considerable between-person variation in phytoestrogen intake in Western populations [30,31]. This suggests that it is methodologically feasible to investigate the effect of phytoestrogens on health and disease in non-Asian countries. Several studies have shown that urinary concentrations of phytoestrogens are reliable, although modest, biomarker of phytoestrogen intake in both Asian and Western populations [11,32-36]. Significant positive correlations have been observed between usual intake of phytoestrogens and their urinary concentrations (e.g. $r=0.54$ for isoflavones and $r=0.40$ for lignans in a Canadian study [33] and r=0.31 for isoflavones in a Hawaii study [34]. Correlations of similar magnitude have also been identified between soy intake and urinary phytoestrogens among Seventh-day Adventists (individuals with a wide range of soy intake) [35] and Minnesota residents [36]. To date, no epidemiologic studies have evaluated the associations between phytoestrogen intake and total cancer, cardiovascular, and all-cause mortality in a nationally representative sample of the U.S. population. Therefore, the present study investigated this research question using data on urinary excretion of total and individual phytoestrogens as well as total cancer, cardiovascular, and all-cause mortality, previously collected from the continuous National Health and Nutrition Examination Survey (NHANES).

\section{Subjects and methods}

Study population

Data analyzed in this study were obtained from the NHANES for the years 1999-2004 and the NHANES linked public-use mortality file. The mortality file was created from a follow-up study of mortality that matched records from the individual years of the NHANES study with data in the National Death Index (NDI) through December 31, 2006 [37]. These data sources were selected because urinary phytoestrogen data for this six-year period only have been linked to mortality data in the NDI. NHANES is a cross-sectional study conducted by the Center for Disease Prevention and Control to assess the health and nutritional status of the general U.S. population. Data collection and sampling procedures for NHANES have been described in detail elsewhere [38]. Sample weights were applied to the data through the calculation of a six-year weight variable according to the guidelines from the National Center for Health Statistics (NCHS) when combining two or more two-year cycles of the continuous NHANES data to produce an unbiased national estimate.

From 1999 to 2004, 29,402 individuals enrolled in the NHANES completed the interview and health examination. As the objective of the present study was to investigate urinary phytoestrogens in relation to cancer, cardiovascular, and all-cause mortality, our analysis was confined to subjects who were $\geq 18$ years and completed a 
24-hour dietary recall, reducing the sample size to 17,061. Urinary concentrations of phytoestrogens were measured among approximately one-third of total NHANES participants. Subsampling in NHANES was performed to reduce participant burden and facilitate scheduling and completion of examinations. All subjects in the subsample were randomly selected from the pool of total participants to obtain a nationally representative sample, with subsample weights calculated to account for probability of being selected into the subsample and additional non-response [39]. Excluding subjects without data on urinary phytoestrogens left the cohort with 5,179 subjects, for whom 79 cancer deaths, 108 cardiovascular deaths, and 290 all-cause deaths were identified during a mean follow up of approximately 5 years (1999-2006). The de-identified data analyzed in the present study are freely available in public domains, and the approval for such data analysis by the Institutional Review Board of Indiana University was sought but determined not to be applicable.

Baseline data collection

NHANES participants were interviewed to collect data on age, sex, race (non-Hispanic white, non-Hispanic black, and other race including multiracial), marital status (married or living with partner, widowed, divorced or separated, and never married), and education level (less than high school, high school graduate or equivalent, and more than high school). Data were also collected on smoking status [never smokers (smoking 0 or $<100$ cigarettes in lifetime), former smokers (smoking $\geq 100$ cigarettes in lifetime but not currently smoking), and current smokers], alcohol consumption ( 0 drink/week, $<1$ drink/week, and $>1$ drink/week), and nutrient intake through a 24-hour food recall. Body mass index (BMI) $\left(\mathrm{kg} / \mathrm{m}^{2}\right)$ was calculated from height and weight measured during the medical examination portion of data collection.

Urinary phytoestrogen measurement

Phytoestrogen biomonitoring was accomplished by measuring urinary excretion of isoflavones (including daidzein, genistein, equol, and O-desmethylangolensin) and enterolignans (including enterodiol, and enterolactone) using high performance liquid chromatography (HPLC) with tandem mass spectrometric (MS/MS) detection [40]. The methods for the collection and analysis of urine samples for phytoestrogen concentrations have been described in detail elsewhere [41]. Briefly, subjects were assigned a date and time to report to one of the Mobile Examination Centers to donate a urine sample. Spot urine specimens were collected the morning after a recommended fast, processed, stored at $-20^{\circ} \mathrm{C}$, and then shipped to the Division of Environmental Health Laboratory Sciences at the NCHS for analysis. Urine samples were amended with stable isotope-labeled internal standards to improve method 
accuracy and precision, incubated with a deconjugation enzyme to allow the quantification of individual phytoestrogens, extracted using solid phase extraction to remove interferences and improve sensitivity, and then analyzed using negative ion mode electrospray ionization HPLC-MS/MS, an assay with a high degree of specificity for each analyte [41].

Mortality follow-up

International Classification of Diseases $10^{\text {th }}$ Revision (ICD-10) codes were used in the selected databases that recorded cause-specific deaths ascertained during follow-up through December 31, 2006 [37]. The underlying causes of death were grouped according to the guidelines provided by the NCHS. The primary outcomes of the present study were cancer mortality (ICD-10 codes, C0-C97), cardiovascular mortality (ICD-10 codes, I00-I99), and all-cause mortality [42].

Statistical analysis

The study population was divided into tertiles based on individuals' urinary concentrations of both total and each individual phytoestrogen to allow for an adequate number of subjects in each group. Total phytoestrogens were calculated by summing up all of the individual phytoestrogens, with a similar calculation completed for both total isoflavones and total enterolignans. Demographic, anthropometric, and lifestyle characteristics of subjects (including age, gender, race, BMI, education, smoking status, and alcohol intake) were compared by the tertiles of total urinary phytoestrogen (ng/ml) (tertile 1: 4 - 414; tertile 2: 415 - 1,047; tertile 3: 1,048 - 112,457). Chi-square tests and analysis of variance were employed to compare differences in categorical and continuous variables among tertiles, respectively. Urinary concentrations of total and individual phytoestrogens were summarized by medians and interquartile ranges. Two-sided t-tests were used to compare them between groups using log-transformed values to account for skewed distributions.

Cox proportional hazards regression was performed to calculate hazard ratios (HRs) and 95\% confidence intervals (CIs) for cancer, cardiovascular, and all-cause mortality in relation to urinary phytoestrogens. Deaths from other causes were treated as censored events in the analyses. The time variable for the Cox models was defined as the time period from the initial NHANES interview date to the date of death or December 31, 2006, whichever occurred first. The lowest tertile of urinary concentrations was the reference group to estimate HRs and 95\% CIs for the two upper tertiles. The multivariable models were adjusted for age, BMI, education, smoking status, total energy intake, sodium intake, and urinary creatinine. Urinary excretion of creatinine was entered into the models to 
account for urine dilution. Gender, race, marital status, and intake of fruits, vegetables, alcohol, fat, and calcium were examined as potential confounders but not included in the final models because they were not significantly associated with any of outcomes of interest in univariate models or did not substantively alter any risk estimates for all outcomes considered $(<10 \%)$. No interactions tested were found to be statistically significant or exhibited clear patterns, and thus no interaction terms were included in the final model. Factors that were tested for their interactions with urinary phytoestrogens in relation to each of the three outcomes included age, gender, BMI, education, smoking status, total energy intake, and sodium intake. Linear trends across tertiles of phytoestrogen intake were tested by using the median in each tertile to create a continuous variable. A two-sided p-value of $<0.05$ was considered statistically significant. SAS version 9.4 (Cary, NC) was used for all statistical analyses.

\section{Results}

Characteristics of study subjects are shown in Table 1. Subjects were statistically significantly different across total phytoestrogen tertiles for gender, race, education, smoking status, and alcohol intake. Those in the highest tertile of urinary phytoestrogens were more likely to be male, non-Hispanic white, have more years of education, and be never smokers, but were less likely to be obese and non-drinkers.

Table 2 shows differences in urinary concentrations of total and individual phytoestrogens between subjects who died of total cancer, cardiovascular disease, and all causes and those who remained alive during follow up through the censor date (December 31, 2006). The median urinary concentrations of total phytoestrogens were lower in individuals who died of each of the three outcomes examined than respective individuals who were alive. Similarly, lower urinary concentrations of total enterolignans were observed for subjects who died of cardiovascular disease and all causes, and lower urinary levels of enterolactone were found for those who died of all causes. Conversely, the median urinary concentrations of total isoflavones and daidzein were higher among individuals who died of cardiovascular disease and all causes than those who remained alive. No significant differences in logtransformed means of total and individual phytoestrogens existed between subjects who did and did not die of each of the three outcomes of interest.

Risk estimates for each of the three outcomes examined in relation to urinary excretion of total and individual phytoestrogens are presented in Table 3. After adjustment for confounders, total phytoestrogens and each of individual phytoestrogens were not associated with a significantly altered risk of death from total cancers. A significantly increased risk of death from cardiovascular disease was found for higher urinary excretion of total 
isoflavones (HR for tertile 3 vs. tertile 1: 2.14; 95\% CI: 1.03, 4.47) and urinary daidzein (HR for tertile 3 vs. tertile 1: 2.05; 95\% CI: 1.02, 4.11). Conversely, higher total enterolignan excretion was significantly associated with a reduced risk of death from cardiovascular disease (HR for tertile 3 vs. tertile 1: 0.48; 95\% CI: 0.24, 0.97). Similarly, a significantly reduced all-cause mortality was found for higher urinary excretion of total enterolignans (HR for tertile 3 vs. tertile 1: 0.65; 95\% CI: 0.43, 0.96) and enterolactone (HR for tertile 3 vs. tertile 1: 0.65; 95\% CI: 0.44 , 0.97). There was a suggestive threshold effect of urinary isoflavones and enterolignan on cardiovascular mortality and urinary isoflavones on all-cause mortality.

To evaluate the possibility of reverse causality arising from preexisting chronic diseases, additional analyses were performed by removing individuals from the dataset who died within two years of enrollment into the study ( $n=24$ for total cancer and $n=43$ for cardiovascular disease) $[43,44]$. An increased risk of cancer death was observed for subjects in the second tertile of urinary total isoflavones (HR: 2.62; 95\% CI: 1.13, 6.10), but risk estimates for all other phytoestrogens remained insignificant. An increased risk of cardiovascular death persisted for subjects in the third tertile of urinary total isoflavones (HR: 2.79; 95\% CI: 1.10, 7.06), but an increased risk for individuals in the third tertile of urinary daidzein and a decreased risk for those in the third tertile of urinary total enterolignans (HR: 0.39; 95\% CI: $0.15,1.00$ ) were no longer significant. The reduced risk of all-cause mortality disappeared for subjects in the third tertile of urinary total enterolignans and the third tertile of urinary enterolactone.

\section{Discussion}

The present study investigated the associations between urinary phytoestrogens and cancer, cardiovascular, and all-cause mortality using data collected from a nationally representative sample of the U.S. population. It was found that urinary concentrations of total enterolignans were significantly and inversely associated with cardiovascular and all-cause mortality, whereas urinary concentrations of total isoflavones and daidzein were significantly and positively associated with cardiovascular mortality. In addition, higher urinary concentrations of enterolactone were significantly associated with lower all-cause mortality.

Genistein is a main isoflavone present in soy products and has been one of the most widely investigated phytoestrogen metabolites. The present study did not show a significant association between urinary genistein and total cancer mortality, which was consistent with the results of several other studies in which genistein intake was not associated with the risk of different types of cancer [23,24,45]. Some studies have reported an inverse association between plasma concentrations of genistein and the risk of prostate and breast cancers [46,47]. A few 
experimental studies revealed a protective effect of genistein on prostate cancer $[48,49]$, whereas another experimental study reported an increased risk of colon cancer associated with genistein intake [50]. Collectively, all the studies discussed above suggest that dietary intake of individual isoflavones or lignans may exert different effects on individual types of cancer. Given the small number of total cancer deaths ( $n=79)$ in the present study, it was not possible to examine cancer-specific associations with total and individual phytoestrogens, an intriguing question worthy of investigation in cohort studies with a larger number of cases of common cancers.

Enterolactone is the main lignan metabolite in both urine and blood [11]. The urinary concentrations of this metabolite were found to reflect the habitual dietary intake of plant lignans [9]. As the precursors of enterolactone are detected in whole-grain products, legumes, seeds, fruits, and vegetables, the urinary concentrations of enterolactone are considered a biomarker for an overall healthy diet [23]. The present study showed low all-cause mortality associated with elevated urinary excretion of both total enterolignans and enterolactone. The consumption of lignan-rich foods has been associated with a decreased risk of breast and prostate cancers in some studies [18] and an increased risk of prostate cancer in other studies [51]. The present study did not show a significant association between urinary excretion of total or individual enterolignans and total cancer mortality. It has been found that enterolactone suppressed the proliferation and migration of prostate cancer cells [52], which suggests that enterolactone intake may reduce the risk of prostate and some other cancers. The differential effects of enterolactone intake on the risk of different sites of cancer [18,51] may account in part for the null results observed for this compound in relation to total cancer mortality in the present study. A significantly reduced risk of cardiovascular death associated with urinary excretion of total enterolignans was observed in the present study, which partially contributes to its inverse association with all-cause mortality.

Experimental and epidemiologic data are scarce examining the influence of intake of total and individual phytoestrogens on cardiovascular health and disease. One study showed that a lignan-rich diet was associated with elevated high-density lipoprotein concentrations and reduced triglyceride concentrations among U.S. adults [53]. Increased serum concentrations of enterolactone have been associated with a reduced risk of acute coronary events and death from cardiovascular disease [54,55]. The results from these previous studies are consistent with those of the present study. This protective effect of enterolactone on cardiovascular disease may be partially attributable to the inverse associations of its high urinary concentrations with inflammation biomarkers (C-reactive protein and white blood cell counts), obesity, and metabolic syndrome in human studies [56-58]. Animal and in vitro studies 
have offered additional mechanistic basis for the reduced cardiovascular mortality associated with elevated levels of urinary enterolactone [59-61]. Specifically, lignan complex [including secoisolariciresinol diglucoside (SDG)] isolated from flaxseed reduced the extent of hypercholesterolemic atherosclerosis and promoted its regression in rabbits [59,60]. SDG induced an elevated expression of vascular endothelial growth factor (VEGF) in human coronary arteriolar endothelial cells [61], and lack of VEGF led to ischemic cardiomyopathy in mice [62].

Additionally, the present study showed an increased risk of cardiovascular death associated with urinary excretion of total isoflavones and daidzein. The results of previous studies on these associations are conflicting. A placebo-controlled, double-blinded trial of postmenopausal women supplemented with isoflavone soy protein showed no statistically significant effect on atherosclerosis progression [63]. Similarly, a meta-analysis of randomized controlled trials revealed that isoflavone supplementation did not improve endothelial function in postmenopausal women with high baseline flow-mediated dilation levels, but significant benefits were found for those with low baseline flow-mediated levels [64]. A cross-sectional study on middle-aged men in the U.S. reported that usual intake of isoflavones was not associated with a favorable cardiovascular risk profile [65]. A protective or null effect of isoflavones on cardiovascular disease that was observed in previous studies was inconsistent with a deleterious effect that was found in the present study. This difference might have arisen from two reasons: 1) most previous studies were small dietary intervention trials among postmenopausal women; 2) in those studies, indicators of cardiovascular functions or biomarkers of cardiovascular lesions were examined; instead, the present study evaluated urinary excretion of total isoflavones and daidzein in relation to cardiovascular mortality among adult women and men of all ages. The potential biological mechanisms for an increased risk of cardiovascular disease associated with urinary levels of isoflavones remain elusive. However, genistein enhanced the gene expression of coagulation factors (prothrombin, factor VII, fibrinogen alpha, and fibrinogen beta) and C-reactive protein (all linked to cardiovascular disease risk) in ovariectomised rats [66].

The present study has several advantages. Exposure to total and individual phytoestrogens was evaluated by measuring their urinary concentrations. Urinary excretion of phytoestrogens is free of recall bias inherent in food frequency questionnaires and is an integrated reflection of phytoestrogen intakes from all sources, including those that may be inadequately represented in food composition databases. For example, the most abundant sources of isoflavones in the diet are from foods containing soy products, such as tofu. However, soy additives are found in some processed foods [67], and certain isoflavones are naturally present in lower concentrations in other foods such 
as vegetables [68], fruits, and nuts [69]. Another theoretical advantage of measuring urinary phytoestrogens is that this assay can also capture phytoestrogen metabolites (e.g. equol and O-desmethylangolensin) produced by intestinal bacteria [70]. It is critical to determine amounts of exposure to specific phytoestrogens because they differ in their levels of biological activity [18]. The US Department of Agriculture has a food composition database for isoflavones but not for lignans [71], which does not allow us to calculate dietary intake of total phytoestrogens for participants in the NHANES. The present study is one of the first studies that used urinary phytoestrogens as biomarkers of their dietary intake to evaluate the effect of these bioactive compounds on the risk of death from cancer and cardiovascular disease. Most previous investigations of the effect of phytoestrogens on cancer risk were small case-control studies [51,72]. Another strength of the present study is that the analysis prospectively evaluated associations between urinary phytoestrogens and all-cause and cause-specific mortality. The data used are based on a nationally representative sample with a relatively large between-person variation in urinary excretion of individual and total phytoestrogens.

Limitations of the present study need to be considered in the interpretation of obtained results. A small number of events for both cancer mortality and cardiovascular mortality did not allow us to perform a stratified analysis by type of cancer or cardiovascular disease. Future studies that incorporate a longer follow-up period may provide new insights into the etiology of cancers and cardiovascular diseases. Lack of adequate power may explain the null associations between urinary phytoestrogens (especially isoflavones) and cancer mortality. Spot urine was used to determine phytoestrogen concentrations, and the results of these measurements might be different from those using 24-hour urine due to potential circadian rhythm. To adjust for urine dilution, phytoestrogen concentrations were normalized to urinary creatinine levels by including urinary creatinine in the Cox models, a commonly used method [32,73], because creatinine is excreted by glomerular filtration at a relatively constant rate [74]. There have been no studies examining the correlation between spot and 24-hour urinary phytoestrogen concentrations. However, the concentrations of phytoestrogens, particularly individual isoflavones, in spot urine have been reported to be statistically significantly correlated with their concentrations measured in serum [75]. In addition, urinary biomarkers of phytoestrogens were measured only once, and a single measurement might not accurately reflect individuals' usual dietary intake due to within-person variation. To capture habitual intake of phytoestrogens, repeated measurements of urinary excretion of this family of chemicals may be necessary, but data on such repeated measurements are not available from NHANES due to feasibility limitations. Therefore, it is possible that some 
subjects might have been misclassified with regard to phytoestrogen intake because of a single measurement of urinary phytoestrogens and their modest correlations with dietary intake.

Significant associations of urinary excretion of daidzein and total enterolignans with cardiovascular and/or allcause mortality disappeared after excluding subjects who died within two years of enrollment, which suggests that these associations reported in Table 3 may be partially ascribed to reverse causality due to the presence of subclinical disease. As NHANES did not exclude individuals with diseases at baseline, some individuals with clinical and/or subclinical disease might have been included in this study. Exact biological or physiological functions of most individual phytoestrogens remain to be elucidated. Therefore, caution needs to be exercised when interpreting their observed effects on disease risk in epidemiologic studies. Mortality data were analyzed in the present study. Therefore, obtained results may be less relevant to the etiology of total cancer and cardiovascular diseases than, and could not be directly compared with, those from analysis of incidence data because mortality of these two outcomes may be influenced by differences in access to and quality of medical treatment among study subjects. No significant differences existed in sex, race, BMI, and smoking status between the participants who donated a urine sample and those who did not. Although the former were a little younger, attained a somewhat higher level of education, and were more likely to drink alcohol than the latter, the differences in these variables were small and the impact was considered inconsequential.

In summary, the present study suggests that higher urinary concentrations of total enterolignans were associated with a reduced risk of death from cardiovascular disease. Similarly, elevated urinary concentrations of both total enterolignans and enterolactone were associated with low all-cause mortality. Conversely, higher urinary concentrations of total isoflavones and daidzein were significantly associated with an increased risk of death from cardiovascular disease and all causes. The observed results of total phytoestrogens need to be interpreted with caution due to potential differences in the physiological functions of individual phytoestrogens. It is important and timely to further investigate the associations of phytoestrogen intake, its biomarkers, and metabolic polymorphisms with the risk of total cancer, specific cancers, and cardiovascular disease in large prospective cohort studies as data generated from such studies may offer innovative avenues for the prevention of these major diseases among people across the world. 
Acknowledgements: There was no specific funding for reported research. 
Conflict of interest: The authors declare that they have no conflict of interest. 
Ethical standards: The de-identified data analyzed in the present study are freely available in public domains, and the approval for such data analysis by the Institutional Review Board of Indiana University was sought but determined not to be applicable.

\section{References}

1. Murphy SL, Xu J, Kochanek KD (2013) Deaths: Final Data for 2010. Centers for Disease Control and Prevention, Atlanta

2. The top 10 causes of death: Fact Sheet Number 310 (2013). World Health Organization,

3. Latest world cancer statistics - Press release number 223 (2013). World Health Organization, Lyon

4. Kuhnle GG, Dell'Aquila C, Low YL, Kussmaul M, Bingham SA (2007) Extraction and quantification of phytoestrogens in foods using automated solid-phase extraction and LC/MS/MS. Anal Chem 79 (23):9234-9239

5. Horn-Ross PL, Barnes S, Lee M, Coward L, Mandel E, Koo K, John EM, Smith M (2000) Assessing phytoestrogen exposure in epidemiologic studies: Development of a database (United States). Cancer Cause Control 11:299-302

6. Adlercreutz H (2007) Lignans and human health. Crit Rev Clin Lab Sci 44:483-525

7. Thomas BF, Zeisel SH, Busby MG, Hill JM, Mitchell RA, Scheffler NM, Brown SS, Bloeden LT, Dix KJ, Jeffcoat AR (2001) Quantitative analysis of the principle soy isoflavones genistein, daidzein and glycitein, and their primary conjugated metabolites in human plasma and urine using reversed-phase high-performance liquid chromatography with ultraviolet detection. J Chromatogr B Biomed Sci Appl 760 (2):191-205

8. Griffiths K, Denis L, Turkes A, Morton MS (1998) Possible relationship between dietary factors and pathogenesis of prostate cancer. Int J Urol 5:195-213

9. Rowland IR, Wiseman H, Sanders TA, Adlercreutz H, Bowey EA (2000) Interindividual variation in metabolism of soy isoflavones and lignans: Influence of habitual diet on equol production by the gut microflora. Nutr Cancer $36: 27-32$

10. Akaza H, Miyanaga N, Takashima N, Naito S, Hirao Y, Tsukamoto T, Mori M (2002) Is daidzein nonmetabolizer a high risk for prostate cancer? A case-controlled study of serum soybean isoflavone concentration. Jpn J Clin Oncol 32 (8):296-300

11. Lampe JW (2003) Isoflavonoid and lignan phytoestrogens as dietary biomarkers. J Nutr 133(Suppl):956S-964S

12. Ohno S, Nakajima Y, Inoue K, Nakazawa H, Nakajin S (2003) Genistein administration decreases serum corticosterone and testosterone levels in rats. Life Sci 74:733-742 
13. Matori H, Umar S, Nadadur RD, Sharma S, Partow-Navid R, Afkhami M, Amjedi M, Eghbali M (2012) Genistein, a soy phytoestrogen, reverses severe pulmonary hypertension and prevents right heart failure in rats. Hypertension 60 (2):425-430

14. Shi L, Ryan HH, Jones E, Simas TA, Lichenstein AH, Sun Q, L HL (2014) Urinary isoflavone concentrations are inversely associated with cardiometabolic risk markers in pregnant U.S. women. J Nutr 144 (3):344-351

15. Nicastro HL, Mondul AM, Rohrmann S, Platz EA (2013) Associations between urinary soy isoflavonoids and two inflammatory markers in the United States in 2005-2008. Cancer Cause Control 24 (6):1185-1196

16. Branham WA, Dial SL, Moland CL, Hass BS, Blair RM, Fang H, Shi L, Tong W, Perkins RG, Sheehan DM (2002) Phytoestrogens and mycoestrogens bind to the rat uterine estrogen receptor. J Nutr 132 (4):658-664

17. Holzbeierlein JM, McIntosh J, Thrasher JB (2005) The role of soy phytoestrogens in prostate cancer. Curr Opin Urol 15:17-22

18. Magee PJ, Rowland IR (2004) Phytoestrogens, their mechanism of action: Current evidence for a role in breast and prostate cancer. Br J Nutr 91:513-531

19. Kuipper GG, Lemmen JG, Carlsson B, Corton JC, Safe SH, van der Saag PT, van der Burg B, Gustafsson JA (1998) Interaction of estrogenic chemicals and phytoestrogens with estrogen receptor beta. Endocrinology 139 (10):4252-4263

20. Onozawa M, Fukuda K, Ohtani M, Akaza H, Sugimura T, Wakabayashi K (1998) Effects of soybean isoflavones on cell growth and apoptosis of the human prostatic cancer cell link LNCaP. Jpn J Clin Oncol 28:360-363

21. Turner JV, Agatonovic-Kustrin S, Glass BD (2007) Molecular aspects of phytoestrogen selective binding at estrogen receptors. J Pharm Sci 96 (8):1879-1885

22. Weber KS, Setchell KD, Stocco DM, Lephart ED (2001) Dietary soy-phytoestrogens decrease testosterone levels and prostate weight without altering LH, prostate 5-alpha-reductase or testicular steroidogenic acute regulatory peptide levels in adult male Sprague-Dawley rats. J Endocrinol 170 (3):591-599

23. Heald CL, Ritchie MR, Bolton-Smith C, Morton MS, Alexander FE (2007) Phyto-oestrogens and risk of prostate cancer in Scottish men. Br J Nutr 98:388-396

24. Hedelin M, Klint A, Chang ET, Bellocco R, Johansson JE, Andersson SO, Heinonen SM, Adlercreutz H, Adami HO, Gronberg H, Balter KA (2006) Dietary phytoestrogen, serum enterolactone and risk of prostate cancer: The cancer prostate Sweden study. Cancer Cause Control 17:169-180 
25. Ziegler RG (2004) Phytoestrogens and breast cancer. Am J Clin Nutr 79 (2):183-184

26. Horn-Ross PL, John EM, Lee M, Stewart SL, Koo J, Sakoda LC, Shiau AC, Goldstein J, Davis P, Perez-Stable EJ (2001) Phytoestrogen consumption and breast cancer risk in a multiethnic population. Am J Epidemiol 154 (5):434-441

27. van der Schouw YT, Kreijkamp-Kaspers S, Peeters PHM, Keinan-Boker L, Rimm EB, Grobbee DE (2005) Cardiovascular Disease in Women: Prospective study on usual dietary phytoestrogen intake and cardiovascular disease risk in western women. Circulation 111 (4):465-471

28. Lissin LW, Cooke JP (2000) Phytoestrogens and cardiovascular health. J Am Coll Cardiol 35 (6):1403-1410

29. Adlercreutz H (2002) Phyto-oestrogens and cancer. Lancet Oncol 3:364-373

30. Anderson LN, Cotterchio M, Boucher BA, Kreiger N (2013) Phytoestrogen intake from foods, during adolescence and adulthood, and risk of breast cancer by estrogen and progesterone receptor tumor subgroup among Ontario women. Int J Cancer 132:1683-1692

31. van der Schouw YT, Kreijkamp-Kaspers S, Peeters PH, Keinan-Boker L, Rimm EB, Grobbee DE (2005) Prospective study on usual dietary phytoestrogen intake and cardiovascular disease risk in western women. Circulation 111:465-471

32. Seow A, Shi CY, Franke AA, Hankin JH, Lee HP, Yu MC (1998) Isoflavonoid levels in spot urine are associated with frequency of dietary soy intake in a population-based sample of middle aged and older Chinese in Singapore. Cancer Epidemiol Biomarkers Prev 7:135-140

33. French MR, Thompson LU, Hawker GA (2007) Validation of a phytoestrogen food frequency questionnaire with urinary concentrations of isoflavones and lignan metabolites in premenopausal women. J Am Coll Nutr 26 (1):76-82

34. Maskarinec G, Singh S, Meng L, Franke AA (1998) Dietary soy intake and urinary isoflavone excretion among women from a multiethnic population. Cancer Epidemiol Biomarkers Prev 7 (7):613-619

35. Jaceldo-Siegl K, Fraser GE, Chan J, Franke A, Sabate J (2008) Validation of soy protein estimates from a foodfrequency questionnaire with repeated 24-h recalls and isoflavonoid excretion in overnight urine in a Western population with a wide range of soy intakes. Am J Clin Nutr 87 (5):1422-1427 
36. Lampe JW, Gustafson DR, Hutchins AM, Martini MC, Li S, Wahala K, Grandits GA, Potter JD, Slavin JL (1999) Urinary isoflavonoid and lignan excresion on a Western diet: Relation to soy, vegetable, and fruit intake. Cancer Epidemiol Biomarkers Prev 8:699-707

37. NHANES (1999-2004) Public-use Linked Mortality Files (2006) (2013). National Health and Nutrition Examination Survey.

38. NHANES 1999-2000 Public Data Release File Documentation (2000). US Department of Health and Human Services, Centers for Disease Control and Prevention, Hyattsville, MD

39. Analytic and Reporting Guidelines: The National Health and Nutrition Examination Survey (NHANES) (2006). Centers for Disease Control and Prevention, Hyattsville, MD

40. Rybak ME, Parker DL, Pfeiffer CM (2008) Determination of urinary phytoestrogens by HPLC-MS/MS: A comparison of atmospheric pressure chemical ionization (APCI) and electrospray ionization (ESI). J Chromatogr B Analyt Technol Biomed Life Sci 861 (1):145-150

41. Parker DL (2004) Division of Laboratory Sciences Laboratory Protocol: Phytoestrogens. National Center for Health Statistics, Hyatsville, MD

42. National Health and Nutrition Examination Survey (1999-2004) Linked Mortality Files (2009). Office of Analysis and Epidemiology, Hyatsville, MD

43. Yu O, Eberg M, Benayoun S, Aprikian A, Barist G, Suissa S, Azoulay L (2014) Use of statins and the risk of death in patients with prostate cancer. J Clin Oncol 32 (1):5-11

44. Van Hemelrijck M, Holmberg L, Garmo M, Hammar N, Walldium G, Binda E, Lambe M, Jungner I (2011) Association between levels of c-reactive protein and leukocytes and cancer: Three repeated measurements in the Swedish AMORIS study. Cancer Epidemiol Biomarkers Prev 20 (3):428-438

45. Ozasa K, Nakao M, Watanabe Y, Hayashi K, Miki T, Mikami K, Mori M, Sakauchi F, Washio M, Ito Y, Suzuki K, Wakai K, Tamakoshi A (2004) Serum phytoestrogens and prostate cancer risk in a nested case-control study among Japanese men. Cancer Sci 95 (1):65-71

46. Kurahashi N, Iwasaki M, Inoue M, Sasazuki S, Tsugane S (2008) Plasma isoflavones and subsequent risk of prostate cancer in a nested case-control study: The Japan Public Health Center. J Clin Oncol 26:5923-5929

47. Verhaus M, van Gils CH, Keinan-Boker L, Grace PB, Bingham SA, Peeters PHM (2007) Plasma phytoestrogens and subsequent breast cancer risk. J Clin Oncol 25 (6):648-655 
48. Bylund A, Zhang J-X, Bergh A, Damber JE, Widmark A, Johnsson A, Adlercreutz H, Aman P, Shepherd MJ, Hallmans G (2000) Rye bran and soy protein delay growth and increase apoptosis of human LNCaP prostate adenocarcinoma in nude mice. Prostate 42:304-314

49. Goetzl MA, Van Veldhuizen PJ, Thrasher JB (2007) Effects of soy phytoestrogens on the prostate. Prostate Cancer Prostatic Dis 10:216-223

50. Rao CV, Wang C-X, Simi B, Lubet R, Kelloff G, Steele V, Reddy BS (1997) Enhancement of experimental colon cancer by genistein. Cancer Res 57:3717-3722

51. Jackson MD, McFarlane-Anderson ND, Simon GA, Bennett FI, Walker SP (2010) Urinary phytoestrogens and risk of prostate cancer in Jamaican men. Cancer Cause Control 21 (12):2249-2257

52. Chen L-H, Fang J, Sun Z, Li H, Wu Y, Denmark-Wahnefried W, Lin X (2009) Enterolactone inhibits insulinlike growth factor-1 receptor signaling in human prostatic carconima PC-3 cells. J Nutr 139 (4):653-659

53. Penalvo JL, Lopez-Romero P (2012) Urinary enterolignan concentrations are positively assoicated with serum HDL cholesterol and negatively associated with serum triglycerides in U.S. adults. J Nutr 142 (4):751-756

54. Vanharanta M, Voutilainen S, Lakka TA, van der Lee M, Adlercreutz H, Salonen JT (1999) Risk of acute coronary events according to serum concentrations of enterolactone: A prospective population-based casecontrol study. Lancet 354 (9196):2112-2115

55. Peterson J, Dwyer J, Adlercreutz H, Scalbert A, Jacques P, McCullough ML (2010) Dietary lignans: Physiology and potential for cardivascular disease risk reduction. Nutr Rev 68 (10):571-603

56. Frankenfeld CL (2014) Cardiometabolic risk factors are associated with high urinary enterolactone concentration, independent of urinary enterodiol concentration and dietary fiber intake in adults. J Nutr 144 (9):1446-1453

57. Eichholzer M, Richard A, Nicastro HL, Platz EA, Linseisen J, Rohrmann S (2014) Urinary lignans and inflammatory markers in the US National Health and Nutrition Examination Survey (NHANES) 1999-2004 and 2005-2008. Cancer Cause Control 25 (3):395-403

58. Struja T, Richard A, Linseisen J, Eichholzer M, Rohrmann S (2014) The association between urinary phytoestrogen excretion and components of the metabolic syndrome in NHANES. Eur J Nutr 53 (6):1371-1381

59. Prasad K (2005) Hypocholesterolemic and antiantherosclerotic effect of flax lignan complex isolated from flaxseed. Atherosclerosis 179 (2):269-275 
60. Prasad K (2008) Regression of hypercholesterolemic atherosclerosis in rabbits by seciosolariciresinol diglucoside isolated from flaxseed. Atherosclerosis 197 (1):34-42

61. Penumathsa SV, Koneru S, Thirunavukkarasu M, Zhan L, Prasad K, Maulik N (2007) Secoisolariciresinol diglucoside: relevance to angiogenesis and cardioprotection against ischemia-reperfusion injury. J Pharmacol Exp Ther 320 (2):951-959

62. Carmeliet P, Ng YS, Nuyens D, Theilmeier G, Brusselmans K, Cornelissen I, Ehler E, Kakkar VV, Stalmans I, Mattot V, Perriard JC, Dewerchin M, Flameng W, Nagy A, Lupu F, Moons L, Collen D, D'Amore PA, Shima DT (1999) Impaired myocardial angiogenesis and ischemic cardiomyopathy in mice lacking the vascular endothelial growth factor isoforms VEGF 164 and VEGF188. Nat Med 5 (5):495-502

63. Hodis HN, Mack WJ, Kono N, Azen SP, Shoupe D, Hwang-Levine J, Petitti D, Whitfield-Maxwell L, Yan M, Franke AA, Selzer RH (2011) Isoflavone soy protein supplementation and atherosclerosis progression in healthy postmenopausal women: A randomized controlled trial. Stroke 42:3168-3175

64. Li S-H, Liu X-X, Bai Y-Y, Wang X-J, Sun K, Chen J-Z, Hui R-T (2010) Effect of oral supplementation on vascular endothelial function in postmenopausal women: A meta-analysis of randomized placebo-controlled trials. Am J Clin Nutr 91 (2):480-486

65. van der Schouw YT, Sampson L, Willett WC, Rimm EB (2005) The usual intake of lignans but not that of isoflavones may be related to cardiovascular risk factors in U.S. men. J Nutr 135 (2):260-266

66. Kelly LA, O'Leary JJ, Seidlova-Wuttke D, Wuttke W, Norris LA (2010) Genistein alters coagulation gene expression in ovariectomised rats treated with phytoestrogens. Thromb Haemostasis 104:1250-1257

67. Liggins J, Mulligan A, Runswick S, Bingham SA (2002) Daidzein and genistein content of cereals. Eur J Clin Nutr 56:961-966

68. Liggins J, Bluck LJ, Runswick S, Atkinson C, Coward WA, Bingham SA (2000) Daidzein and genistein contents of vegetables. Br J Nutr 84:717-725

69. Liggins J, Bluck LJ, Runswick S, Atkinson C, Coward WA, Bingham SA (2000) Daidzein and genistein content of fruits and nuts. J Nutr Biochem 11 (6):326-331

70. Rowland I, Faughnan M, Hoey L, Wahala K, Williamson G, Cassidy A (2003) Bioavailability of phytooestrogens. Br J Nutr 89:S45-S58 
71. Bhaqwat S, Haytowitz DB, Holden JM (2008) USDA Database for the Isoflavone Content of Selected Foods. vol 2.0. Nutrient Data Laboratory, Beltsville, MD

72. Park SY, Wilkens LR, Franke AA, Le Marchand L, Kakazu KK, Goodman MT, Murphy SP, Henderson BE, Kolonel LN (2009) Urinary phytoestrogen excretion and prostate cancer risk: A nested case-control study in the Multiethnic Cohort. Br J Cancer 101 (1):185-191

73. Atkinson C, Skor HE, Fitzgibbons ED, Scholes D, Chen C, Wahala K, Schwartz SM, Lampe JW (2002)

Overnight urinary isoflavone excretion in a population of women living in the United States, and its relationship to isoflavone intake. Cancer Epidemiol Biomarkers Prev 11:253-260

74. Barr DB, Wilder LC, Caudill SP, Gonzalez AJ, Needham LL, Pirkle JL (2005) Urinary creatinine concentrations in the U.S. population: Implications for urinary biologic monitoring measurements. Environ Health Perspect 113 (2):192-200

75. Grace PB, Taylor JI, Low Y-L, Luben RN, Mulligan AA, Botting NP, Dowsett M, Welch AA, Khaw K-T, Wareham NJ, Day NE, Bingham SA (2004) Phytoestrogen concentrations in serum and spot urine as biomarkers for dietary phytoestrogen intake and their relation to breast cancer risk in European Prospective Investigation of Cancer and Nutrition-Norfolk. Cancer Epidemiol Biomarkers Prev 13 (5):698-708 
Table 1. Baseline characteristics of subjects by tertiles of urinary concentrations of total phytoestrogens (ng/mL) in the continuous National Health and Nutrition Examination Survey, 1999-2004

\begin{tabular}{|c|c|c|c|c|}
\hline Characteristics & $\begin{array}{l}\text { Tertile } 1 \\
(4-414)\end{array}$ & $\begin{array}{c}\text { Tertile } 2 \\
(415-1,047)\end{array}$ & $\begin{array}{c}\text { Tertile } 3 \\
(1,048-112,457)\end{array}$ & \\
\hline & $\mathrm{n}=1,726$ & $\mathrm{n}=1,727$ & $\mathrm{n}=1,726$ & p-value \\
\hline Age [Mean (SD)] & 44.7 (16.8) & 45.5 (17.9) & $44.8(17.4)$ & 0.28 \\
\hline \multicolumn{5}{|l|}{ Gender (\%) } \\
\hline Male & 45.9 & 47.2 & 51.0 & 0.006 \\
\hline Female & 54.1 & 52.8 & 49.0 & \\
\hline \multicolumn{5}{|l|}{ Race/Ethnicity (\%) } \\
\hline Non-Hispanic White & 70.7 & 71.2 & 72.5 & 0.029 \\
\hline Non-Hispanic Black & 9.9 & 11.8 & 11.7 & \\
\hline Other & 19.4 & 17.0 & 15.8 & \\
\hline BMI [Mean (SD)] & $28.3(6.4)$ & $28.2(5.9)$ & $27.7(6.4)$ & 0.004 \\
\hline \multicolumn{5}{|l|}{ Education (\%) } \\
\hline Less than High School & 23.1 & 20.7 & 19.5 & $<0.001$ \\
\hline \multicolumn{5}{|l|}{ High School Graduate } \\
\hline \multicolumn{5}{|l|}{ or Equivalent } \\
\hline More than High School & 49.6 & 51.8 & 57.6 & \\
\hline \multicolumn{5}{|l|}{ Smoking Status (\%) } \\
\hline Never Smoker & 48.2 & 51.4 & 53.6 & $<0.001$ \\
\hline Former Smoker & 22.9 & 24.1 & 25.3 & \\
\hline Current Smoker & 28.9 & 24.5 & 21.1 & \\
\hline \multicolumn{5}{|l|}{ Alcohol Intake (\%) } \\
\hline 0 drinks/week & 20.2 & 21.0 & 16.7 & 0.025 \\
\hline$<1$ drinks/week & 41.9 & 42.9 & 46.1 & \\
\hline$>1$ drinks/week & 37.9 & 36.2 & 37.3 & \\
\hline
\end{tabular}


Table 2. Differences in urinary concentrations of total and individual phytoestrogens (ng/mL) between subjects who did and did not die of total cancer, cardiovascular disease, or all-causes in the continuous National Health and Nutrition Examination Survey, 1999-2004

\begin{tabular}{|c|c|c|c|c|c|c|}
\hline & \multicolumn{2}{|c|}{ Total Cancer } & \multicolumn{2}{|c|}{ Cardiovascular Diseases } & \multicolumn{2}{|c|}{ All Causes } \\
\hline - & Death & Survival & Death & Survival & Death & Survival \\
\hline Phytoestrogens & $(\mathrm{n}=79)$ & $(\mathrm{n}=5,100)$ & $(n=108)$ & $(\mathrm{n}=5,071)$ & $(n=290)$ & $(\mathrm{n}=4,889)$ \\
\hline Total Phytoestrogen & $607(416,1311)$ & $679(306,1440)$ & $437(268,1083)$ & $682(308,1442)$ & $531(294,1117)$ & $687(308,1453)$ \\
\hline Genistein & $32(13,88)$ & $26(9,89)$ & $28(13,79)$ & $26(9,90)$ & $31(12,79)$ & $26(9,90)$ \\
\hline Daidzein & $78(28,170)$ & $56(18,191)$ & $84(32,143)$ & $56(18,191)$ & $68(21,167)$ & $56(18,191)$ \\
\hline Equol & $8(3,19)$ & $8(2,17)$ & $6(3,14)$ & $8(2,17)$ & $7(3,18)$ & $8(2,17)$ \\
\hline O-desmethylangolensin & $3(0,16)$ & $4(1,19)$ & $5(1,21)$ & $4(1,19)$ & $3(1,16)$ & $4(1,19)$ \\
\hline Enterolignan & $437(213,809)$ & $415(148,928)$ & $299(124,706)$ & $416(149,931)$ & $347(152,750)$ & $417(148,940)$ \\
\hline Enterodiol & $53(18,112)$ & $39(14,92)$ & $32(16,66)$ & $40(14,93)$ & $33(15,86)$ & $40(14,93)$ \\
\hline Enterolactone & $371(171,743)$ & $347(104,821)$ & $240(75,622)$ & $349(105,824)$ & $289(124,628)$ & $351(104,825)$ \\
\hline
\end{tabular}

${ }^{\text {a }}$ Values are medians (interquartile ranges). 


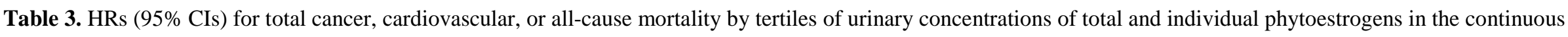
National Health and Nutrition Examination Survey, 1999-2004

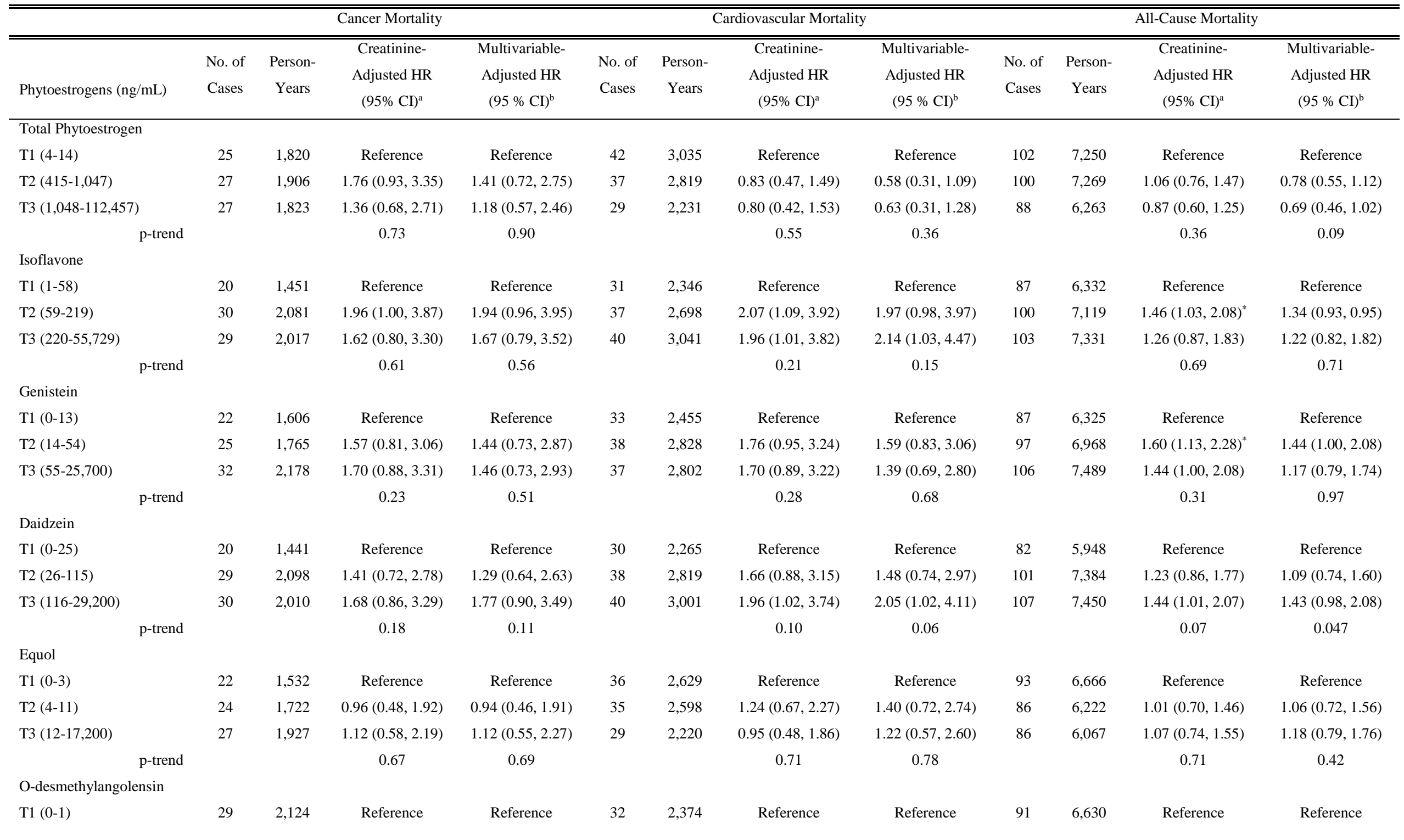




\begin{tabular}{|c|c|c|c|c|c|c|c|c|c|c|c|c|c|}
\hline T2 (2-9) & & 25 & 1,730 & $0.91(0.49,1.71)$ & $0.78(0.41,1.48)$ & 28 & 2,066 & $1.15(0.60,2.19)$ & $1.07(0.53,2.15)$ & 91 & 6,375 & $1.24(0.87,1.77)$ & $1.12(0.77-1.62)$ \\
\hline T3 $(10-9,890)$ & & 23 & 1,569 & $0.83(0.44,1.56)$ & $0.75(0.38,1.48)$ & 40 & 3,025 & $1.50(0.81,2.77)$ & $1.71(0.87,3.35)$ & 93 & 6,725 & $1.13(0.79,1.63)$ & $1.12(0.76,1.65)$ \\
\hline & p-trend & & & 0.59 & 0.58 & & & 0.19 & 0.07 & & & 0.90 & 0.72 \\
\hline \multicolumn{14}{|l|}{ Enterolignan } \\
\hline $\mathrm{T} 1$ (0-225) & & 27 & 1,999 & Reference & Reference & 40 & 2,858 & Reference & Reference & 101 & 7,106 & Reference & Reference \\
\hline T2 (226-691) & & 30 & 2,116 & $1.68(0.90,3.13)$ & $1.43(0.75,2.73)$ & 39 & 3,009 & $0.83(0.47,1.46)$ & $0.55(0.30,1.02)$ & 112 & 8,281 & $1.26(0.91,1.74)$ & $0.99(0.70,1.40)$ \\
\hline Т3 (692-85,847) & & 22 & 1,434 & $1.22(0.62,2.39)$ & $1.05(0.52 .2 .14)$ & 29 & 2,218 & $0.73(0.39,1.38)$ & $0.48(0.24,0.97)$ & 77 & 5,395 & $0.86(0.60,1.25)$ & $0.65(0.43,0.96)$ \\
\hline & p-trend & & & 0.087 & 0.86 & & & 0.36 & 0.07 & & & 0.26 & 0.019 \\
\hline \multicolumn{14}{|l|}{ Enterodiol } \\
\hline $\mathrm{T} 1(0-20)$ & & 27 & 1,991 & Reference & Reference & 38 & 2,805 & Reference & Reference & 105 & 7,566 & Reference & Reference \\
\hline T2 (21-63) & & 22 & 1,586 & $0.94(0.47,1.88)$ & $1.09(0.54,2.22)$ & 38 & 2,961 & $1.15(0.65,2.04)$ & $1.36(0.74,2.48)$ & 93 & 6,877 & $0.92(0.65,1.29)$ & $1.05(0.73,1.50)$ \\
\hline Т3 $(64-18,000)$ & & 28 & 1,835 & $1.60(0.85,3.01)$ & $1.66(0.85,3.34)$ & 30 & 2,177 & $0.92(0.48,1.77)$ & $0.71(0.87,1.78)$ & 88 & 6,060 & $0.97(0.68,1.37)$ & $0.98(0.67,1.43)$ \\
\hline & p-trend & & & 0.08 & 0.10 & & & 0.69 & 0.52 & & & 0.95 & 0.85 \\
\hline \multicolumn{14}{|l|}{ Enterolactone } \\
\hline T1 (0-173) & & 25 & 1,790 & Reference & Reference & 40 & 2,880 & Reference & Reference & 102 & 7,177 & Reference & Reference \\
\hline T3 $(596-85,300)$ & & 22 & 1,429 & $1.19(0.60,2.32)$ & $1.01(0.50,2.05)$ & 31 & 2,368 & $0.78(0.41,1.48)$ & $0.54(0.27,1.07)$ & 78 & 5,464 & $0.86(0.59,1.25)$ & $0.65(0.44,0.97)$ \\
\hline & p-trend & & & 0.99 & 0.72 & & & 0.43 & 0.10 & & & 0.22 & 0.014 \\
\hline
\end{tabular}

HR, hazard ratio; CI, confidence interval.

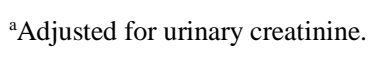

bAdjusted for age, education, smoking status, body mass index, total energy intake, sodium intake, and urinary creatinine. 